\title{
The Performance Prediction of Elite Male Trampolines Based on Physical Fitness Factors
}

\author{
Izzet Uçan \\ Correspondence: Izzet Uçan, School of Physical Education and Sport Sciences, Bayburt University, Turkey.
}

Received: January 25, 2018

doi:10.11114/jets.v6i4a.3259

\author{
Accepted: May 4, 2018 Online Published: May 4, 2018 \\ URL: https://doi.org/10.11114/jets.v6i4a.3259
}

\begin{abstract}
The main purpose of this study was prediction of elite male trampolines performance based on the selected physical capabilities in different age categories. To this aim, 45 male athletes (age 14.17 \pm 2.86 yrs, athletic experience 7.22 \pm 3.64 yrs and competitive experience 3.31 $\pm 2.44 \mathrm{yrs}$ ), who participated in the national trampoline team's preparation camps for participation at the 2014 Asian Games in four age categories included children (ages 11 and 12 yrs), juniors (ages 13 and $14 \mathrm{yrs}$ ), adolescents (ages 15 to $17 \mathrm{yrs}$ ), and seniors (ages 18 yrs <), were selected using targeted sampling. Variables of study included 20 physical fitness factors which were measured using standard procedures and instruments. Data were analyzed using Pearson correlation coefficient and multiple regression models at the 0.05 significance level. Results of study showed that only the relationships of the shoulder belt muscle strength $(r=0.294)$, aerobic power $(r=0.351)$, relative minimum anaerobic power $(r=0.256)$, with performance are statistically significant and in other cases, observed relationships were not statistically significant $(p>0.05)$. Findings of this study suggest that, in the high competitive level of trampoline, physical factors have predictive role in preparation programs of elite male trampolines.
\end{abstract}

Keywords: physical fitness, trampoline, elite athlete

\section{Introduction}

Years of research in the field of championship sport show that successful competition requires a set of specific psychological, physiological, physical fitness, anthropometric and morphological factors that provide profiles of physical fitness of elite athletes in various sports fields support this claim. In the field of gymnastics, trampolines is as one of the most popular sports fields, is not an exception to these psychological and physical requirements, and the achievement of the championship requires these needs.

Recent research results in gymnastics show that, although anthropometric features are important determinants of gymnastic performance, physiological functions play a significant role in this regard (Douda et al., 2016). Nowadays, the presence of various sciences along with the championship sport is a necessary preparation for athletes in sports competitions.

Scientific efforts in the field of sports develop educators' and athletes knowledge and enhance performance in the field of championship. In a comparison between the results of the research can be concluded that, depending on the nature and needs of each sport, various physiological, physical and psychological factors play different roles in different sports disciplines, which emphasize the importance of specific reviews focus on each discipline. However, according to a researcher's search, only two studies have been published on the trampoline field. In this study, Gomez-Lndrv et al (2010) studied two age groups under 15 and over 15 years offering fitness and body composition profile of the Spanish elite women's trampoline workers. In general, the scientific information supplied by the preceding studies on physical capabilities provided trampoline athletes is very limited and it emphasizes the need for more studies in this field.

Accordingly, the results of this study will provide valuable information about the factors involved in physical and mental preparation to educators, athletes and psychologists in this field. On the other hand, examining the physical and mental abilities of the elite trampolines provides an overview of these capabilities that can provide some of the information needed in the process of scouting.

Faria and Faria (2012) studied the relationship between the physical characteristics of young elite male gymnasts and their competitive performance. Based on the results, gymnasts of the first class had a shorter stature, more relative strength and absolute strength, more shoulder flexion, lower back and pelvic, higher muscle mass and lower percentage of fat. Lindner, Caine \& Johns (2013) examined the physical and functional predictors of competitive withdrawal in 
female gymnasiums. The results of the study showed that dropout gymnasts are older, taller and weigh heavier, which were the reason for their higher strength, power, speed and endurance. Discontinued gymnasts with less skinny physics/ectomorphic with less muscle, which had better recordings in most of the flexibility tests.

The exclusive gymnastic flexibility test was the only factor to distinguish gymnasts performance involved in competitions. According to their observations, they concluded that the factors of age, social and psychological factors are distinictive factors between retired and competitive gymnastics.

Hume et al (2014) studied the physical factors determining progress in rhythmic gymnastics. Based on the results, the best correlates of the progress of gymnasts were cumulative training time and training time $(r=-0.530 .84)$. Also, age and muscle mass and body composition, flexibility, lower limb strength, visual-motor performance had significant relationships with the level of progression $(r=-0.29)$.

Dashti (2015) studied the relationship between anthropometric characteristics and selected physical fitness factors with the performance of gymnasts of Iranian male boys. The results of this study showed that variables such as height, length of two hands, fat weight, body weight without fat, finger power and power have a significant relationship with the performance of primary gymnasts. However, there was no significant relationship between the width of shoulder, hip width, hip length, percentage of fat and pelvic flexibility.

Douda et al. (2016) identified the physiological and anthropometric predictors of gymnastics rhythmic performance. The results showed anthropometric components $45 \%$, flexibility $12.8 \%$, explosive power $9.2 \%$, aerobic power $7.4 \%$, body dimensions $6.8 \%$ and anaerobic consumption $4.8 \%$, anthropometric features $(\mathrm{r}=0.5)$ and aerobic power $(\mathrm{r}=0.49)$ had significant correlation with performance.

The results of regression analysis showed that hip circumference and body mass index explained $13.1 \%$ and $8.5 \%$ of variance, respectively. Therefore, anthropometric, aerobic, flexural and explosive power characteristics were reported as important determinants of successful performance.

\section{Materials and Methods}

Based on the method, the present study is predictive correlation research and in terms of purpose, it is prospective one.

\subsection{Society, Sample and Sampling Method}

The statistical population of the study was all male trampolines who were invited to the Islamic Republic of Iran gymnastics training camps in 2014 to participate in Asian Games 2014 and participated in competing selections within the campus.

In a more general division, 48 athletes were invited to the national team camps in four age groups; children (11 and 12 years old), adolescents (13 and 14 years of age), youth (15-17 years) and adults (18 years) (In each age group 12 people). Due to the limited number of people in the statistical population, non-random sampling method was used. As a result, the research sample was equal to the statistical society. However, 3 people in camps were unable to complete measurements due to injury, which dropped out of the sample and the number of samples dropped to 45 . All participants, with full knowledge of the goals and implementation process, participated in this study with full satisfaction.

\subsection{Measuring Tools and Equipment}

In the present study, the following tools and equipment were used to measure the variables of the research:

1. Researcher-made questionnaire for collecting individual information of subjects

2. Digital scales with accuracy of $0.01 \mathrm{~kg}$ equipped with a precision 0.005 meter with SOEHNLE® marking Made in Germany to measure the height and weight of subjects

3. Horizontal stretching test for measuring muscle strength of shoulder belt in subjects

4. Abdominal examination on the Swedish ladder to measure the abdominal strength of the subjects

5. Specific test of angle maintenance on the parallel to measure the muscular endurance of the subjects

6. Standard shoulder flexion (extension and flexion) tests for assessing the joint flexion of the subjects

7. Standard trunk flexural test for measuring the flexural strength of the front trunk

8. Standard trunk flexural test for measuring trunk flexion to the back of the subjects

9. Monarch Aerobic test on Treadmill with TechnoGym® Brand made in Italy to Test Aerobic Exercise

10. Vertical jump and jump tests to test the subjects' explosive power

11. Monarock's Tissue Test on a TechnoGym ${ }^{\circledR}$ Marker Bike made in Italy to measure the muscle strength of the upper body of the subjects 
12. Argus test for 15 seconds to measure the muscle strength of the lower body of the subjects.

13. Zigzag test for 30 seconds to test the agility of the subjects

14. Test of the time of photo-observation in 8 directions

15. Test of the 8-way star movement for measuring the dynamic balance of the subjects

16. Arbitration Board and Arbitration Rules of the Islamic Gymnastics Federation to assess the performance of the subjects

\section{Measurements}

\subsection{Personal Information}

Participants' individual information was compiled by using a researcher-made questionnaire consisting of eight questions for determining age, competitive age, sport record, competitive history, history of membership in the national team, weekly physical and mental training hours and the records of the championship.

\subsection{Physical Fitness Factors}

The tests used to measure physical-motor capabilities are described in Table 1-3. It needs to be explained that in the tests of flexibility, explosive power, bleeding power and equilibrium, the requirements of the participants' records were normalized, described in table 3. Also, the correct implementation of the tests was supervised by cooperation of Bayburt university experts in Iran's university during the implementation of the test.

\subsection{Competitive Performance in the Implementation of Trampolines}

In order to measure the level of performance of the participants, information about the selection of the camp that was carried out according to the laws of the Islamic gymnastics federation of the Islamic Republic was used. Based on this intra-camp competition, each participant executed 20 trampoline skills and assessed the quality of performance by five national referees.

The range of scores provided by each referee is between 0 and 20 , which is based on the qualitative analysis parameters of skill implementation for each participant. In calculating the performance score, at first, the minimum and maximum grades are eliminated from the five points given for each skill and the average of the three remaining scores is calculated.

Participants were also asked to respond to the questions with integrity and precision, given that there was no right or wrong answer in the questionnaires. At the end of the coordination session, the recommendations were provided by the researcher to the participants about the amount of sleep, nutrition and rest. Then, after coordinating with the physical fitness laboratory of Bayburt University, a body composition measure was performed. At the first session, body composition was measured.

During each session, participants were asked to run a standard warm up protocol that was led by the coaches of trampoline national team for 10 minutes. Before each test, the correct implementation, error criteria and the evaluation of the implementation were explained by the laboratory experts and. Participants were allowed to review their implementation practices before performing the main tests. To prevent fatigue, a rest time test was considered sufficiently. Each measurement session was terminated with a standard cooling protocol. Finally, information about the level of trampoline performance at the end of the training camp was received from the technical team members.

At the end of the coordination session, the recommendations were provided by the researcher to the participants about the amount of sleep, nutrition and rest.

Then, after coordination with the physical fitness abilities laboratory of Bayburt University, measurements on body composition and physical fitness factors were performed. Muscle strength and muscle strength tests were performed in one session.

\section{Data Analysis Method}

In the data analysis, considering the prediction of the natural distribution of the data, Pearson correlation coefficient was used. All analyzes were performed at 95\% confidence level using Excel software and SPSS version 21. 


\section{Results}

Table 1. Descriptive of statistics relating to the individual characteristics of the participants

\begin{tabular}{|c|c|c|c|c|}
\hline & $\mathrm{N}$ & $\bar{M}$ & & SD \\
\hline Age (years) & 45 & & 14.17 & 2.86 \\
\hline Sport record (years) & 45 & & 7.22 & 3.64 \\
\hline Competition (years) & 45 & & 3.31 & 2.44 \\
\hline
\end{tabular}

Individual characteristics in Table 1 show that age, sport history and competitive history of the participants in the study.

Table 2. Distribution of participants based on selected sport variables

\begin{tabular}{lllr}
\hline Characteristic & Subcategory & \multicolumn{2}{c}{ Statistical indices } \\
\hline Competitive age & & $\mathrm{N}$ & $\%$ \\
& L5 (born in 2001) & 8 & $\% 17.8$ \\
& L6 (born in 2000) & 7 & $\% 15.6$ \\
& L7 (born in 1999) & 8 & $\% 17.8$ \\
& L8 (born in 1998) & 4 & $\% 8.9$ \\
History of membership in the national team & Youth (born 1996-97) & 6 & $\% .13 .3$ \\
& Adults (born in 1995 and below) & 12 & $\% 26.7$ \\
& Y & 19 & $\% 42.2$ \\
& $\mathrm{~N}$ & 26 & $\% 52.8$ \\
\hline
\end{tabular}

Table 2 indicates the age category of competitive groups.

Table 3. Descriptive statistics of physical fitness factors

\begin{tabular}{lrrr}
\hline & Indices & & \\
& $\mathrm{N}$ & $\mathrm{M}$ & $\mathrm{SD}$ \\
\hline Shoulder muscle strength (maximum repetition) & 45 & 10.97 & 3.96 \\
Abdominal muscle strength (Maximum repeat) & 45 & 6.30 & 4.70 \\
Muscle endurance (s) & 45 & 16.55 & 9.38 \\
Shoulder joint flexion - upward (\% hand length) & 45 & 51.59 & 13.43 \\
Shoulder Joint Flexibility - Backward (\% Hand Length) & 45 & 74.16 & 7.09 \\
Flexible forward trunk (cm) & 45 & 14.95 & 4.66 \\
Flexural trunk to the back (\% sit tall) & 45 & 60.86 & 7.74 \\
Aerobic power (ml / kg per minute) & 45 & 42.30 & 5.40 \\
Explosive Power - Vertical Jump Record (\% Length) & 45 & 27.58 & 10.14 \\
Explosive Power - Record Jumping Length (\% Length) & 45 & 123.85 & 11.25 \\
Bleeding the upper muscles of the trunk & 45 & - & - \\
Maximum Absolute Bulking Capacity (W) & 45 & 499.47 & 224.31 \\
Absolute Average Bleeding Power (W) & 45 & 347.90 & 150.37 \\
Absolute Power (Watts) & 45 & 194.71 & 102.98 \\
Maximum Relative Expansion Power (watts per kilogram) & 45 & 9.84 & 2.02 \\
Relative mean bleeding power (watts per kilogram) & 45 & 6.85 & 1.24 \\
Minimal Relative Potency (Watts per Kilogram) & 45 & 3.78 & 1.32 \\
Bleeding power of the lower muscles of the trunk (watts per kilogram) & 45 & 42.88 & 7.44 \\
Agility - zigzag test record (repeat in 30 seconds) & 45 & 60.11 & 8.52 \\
Viewing time (watts per kilogram) & 45 & 10.37 & 2.07 \\
Dynamic Balance (\% Foot Length) & 45 & 79.91 & 6.32 \\
Dynamic balance on the right leg (\% foot length) & 45 & 83.01 & 7.18 \\
Dynamic balance on the left foot (\% leg length) & 45 & 76.82 & 6.44 \\
Dynamic balance difference of right and left foot (\% leg length) & 45 & 6.68 & 4.43 \\
\hline
\end{tabular}


Table 4. Results of Pearson correlation coefficient to determine the relationship between physical fitness factors and competitive performance

\begin{tabular}{lrrr}
\hline Predictive variables & N & \multicolumn{1}{l}{ R } & \multicolumn{1}{l}{ P } \\
\hline The muscle strength of the shoulder belt & 45 & 0.294 & $0.05^{*}$ \\
Abdominal muscle strength & 45 & 0.248 & 0.118 \\
Muscle endurance & 45 & -0.029 & 0.868 \\
Shoulder Joint Flexibility - Upward & 45 & 0.162 & 0.312 \\
Shoulder joint flexion - Back to back & 45 & -0.096 & 0.550 \\
Flexural forward trunk & 45 & 0.104 & 0.517 \\
Thoracic torso flexion & 45 & 0.164 & 0.304 \\
Aerobic power & 45 & 0.351 & $0.047^{*}$ \\
Explosive Power - Vertical Jump Record & 45 & 0.02 & 0.901 \\
Explosive Power - Record Long Jump & 45 & 0.057 & 0.725 \\
Bleeding the muscles of the upper trunk & 45 & - & - \\
Maximum Relative Absolute Power & 45 & 0.057 & 0.731 \\
Medium Relative Bleeding Possibility & 45 & 0.094 & 0.569 \\
Minimal bleeding power & 45 & 0.256 & $0.05{ }^{*}$ \\
Bleeding the muscles of the lower trunk & 45 & 0.207 & 0.194 \\
Agility - Zigzag test record & 45 & 0.011 & 0.947 \\
Observation of Response Time & 45 & -0.08 & 0.617 \\
Dynamic balance & 45 & 0.082 & 0.613 \\
Dynamic balance on the right foot & 45 & 0.084 & 0.606 \\
Dynamic balance on the left foot & 45 & 0.068 & 0.675 \\
Dynamic balance difference between right and left legs & 45 & 0.017 & 0.915 \\
The muscle strength of the shoulder belt & 45 & 0.284 & $0.05{ }^{*}$ \\
\hline
\end{tabular}

The results of Pearson correlation coefficients in table 5 show that the relationship between the performance of elite male trampoliners with muscle strength, shoulder muscle strength, aerobic power, and minimum relative biopsy power is statistically significant, but there was no relationship between performance with abdominal muscle strength muscular endurance, shoulder joint flexibility-upward, shoulder joint flexibility-backward, forward trunk flexibility, trunk flexural strength, explosive power-vertical jump, explosive power-jump length, maximum relative tensile strength, high tympanic muscle, relative mean muscle tone of upper muscle, lower muscle tone, agility-zigzag test record. The observation image, dynamic balance, dynamic balance on the right foot, dynamic balance on the left leg and the dynamic balance difference between the right and left legs are not statistically significant.

Observed relationships indicate that high levels of performance in elite male trampolines are associated with high levels of shoulder muscle strength, aerobic power, and minimal relative healing power. It can be concluded that there is a significant relationship between some of the factors of physical fitness (muscle strength of shoulder belt, aerobic power and minimum relative bias) and the performance of male elite trampolines.

Table 5. Results of regression model of competitive performance level of elite male trampolines

\begin{tabular}{|c|c|c|c|c|c|c|c|c|c|c|}
\hline $\begin{array}{l}\text { Source of } \\
\text { changes }\end{array}$ & variables entered in model & $\mathrm{df}$ & $\mathrm{R}$ & $\mathrm{R}^{2}$ & $\begin{array}{l}\text { Modified } \\
\mathrm{R}^{2}\end{array}$ & $F$ & $p$ & $R^{2} \Delta$ & $F \Delta$ & $\begin{array}{l}\text { P for } \\
\text { change }\end{array}$ \\
\hline & $\begin{array}{l}\text { Physical fitness factors 1) } \\
\text { Shoulder muscle strength }\end{array}$ & 3 & 0.31 & 0.096 & 0.023 & 1.31 & 0.284 & 0.096 & 1.31 & 0.284 \\
\hline & 2) Aerobic power & & & & & & & & & \\
\hline & $\begin{array}{l}\text { 3) Minimal } \\
\text { Bonding Power }\end{array}$ & & & & & & & & & \\
\hline
\end{tabular}

* It is meaningful at level $0.05 \mathrm{p}$

Contradiction results 5 shows that the level of competitive performance of elite male trampolines is significant in the strength of shoulder muscle strength, aerobic power, and minimal bleeding ability.

\section{Discussion and Conclusion}

The results of the research on physical fitness factors indicated there was a significant relationship between aerobic power, shoulder muscle strength and upper trunk muscle tibial power (minimum relative retardation capacity) with the performance of trampoline elite male athletes and other factors of physical fitness do not have a significant relationship with the performance level of these athletes. These findings suggest that higher aerobic power and higher bleeding power and strength in the upper trunk are associated with higher levels of performance in the trampolines. However, the 
trampoline field is considered as an anaerobic field due to the nature of the motion and the duration of its routines, but nevertheless, the importance of aerobic power as a feature of the vastly large volume of infrastructure athletes' workout and the impact of cardio-pulmonary and circulatory systems can be considered in the recycling process.

Similar findings have also been reported on the importance of aerobic power in the field of gymnastics. For example, Douda et al. (2008) reported a relationship between the aerobic power and the performance of artistic gymnasts $(r=0.49)$, which is expected to be comparable with the trampoline's motion (less time to run). There is a weaker relation between aerobic power and performance in this field than in the field of gymnastics. The findings of this study also indicate the existence of such a relationship $(r=0.351)$.

On the other hand, the meaningful relationship between shoulder muscle strength and high muscle strength of upper trunk with level of function emphasizes the importance of the role of upper muscles of trunk in creating the momentum needed to run the skills of the trampoline, because the skill of those with a higher degree of difficulty in this field require the forward and backward rotation, which requires high muscular strength and muscle strength in the upper trunk. Although the existence of such meaningful relationships determines the factors involved in the exercise performance, the predictive power of these variables should be confirmed on the basis of regression analysis.

In this regard, the results of regression analysis of the level of competitive performance based on the selected factors of physical fitness (muscle strength of shoulder belt, aerobic power and minimum relative power of biosynthesis) showed that these factors cannot predict the level of performance and only $9.6 \%$ of the performance of elite male trampolines is related to these variables. Douda et al. (2008) reported that the contribution of the flexibility factor to gymnastics performance was $12.1 \%$, explosive power $9.2 \%$, aerobic power $4.7 \%$, and fuel metabolism $4.6 \%$, which does not match by finding of the present study. The reason for the inconsistency may be related to the difference in the nature of the two competitive disciplines and the gender difference and the competitive level of the samples. In general, according to the findings of the present study about the factors of physical fitness, only the factors of aerobic capacity, strength and power of the muscles of the upper trunk were related to the performance of elite males.

\section{References}

Dashti, K., \& Mohammad, H. (2015). Relationship between some anthropometric characteristics and physical fitness factors with the performance of gymnasts in the superior male boys. Master's thesis, University of Guilan, Faculty of Physical Education and Sport Sciences.

Douda, H. T., Toubekis, A. G., Avloniti A. A., \& Tokmakidi, S. P. (2016). Physiological and anthropometric determinants of rhythmic gymnastics performance. International Journal of Sport Physiology and Performance, 3, 41-45. https://doi.org/10.1123/ijspp.3.1.41

Faria, I. E., \& Faria, E. W. (2012). Relationship of the anthropometric and physical characteristics of male junior gymnasts to performance. J Sports Med Phys Fitness, 29(4), 369-378.

Hume, P. A., Hopkins, W. G., Robinson, D. M., Robinson, S. M., \& Hollings, S. C. (2014). Predictors of attainment in rhythmic sportive gymnastics. J. Sports Med. Phys. Fitness, 33(4), 367- 377.

Lindner, K. J., Caine, D. J., \& Johns, D. P. (2013). Withdrawal predictors among physical and performance characteristics of female competitive gymnasts. J. Sports Sci., 9(3), 259-272.

https://doi.org/10.1080/02640419108729888

\section{Copyrights}

Copyright for this article is retained by the author(s), with first publication rights granted to the journal.

This is an open-access article distributed under the terms and conditions of the Creative Commons Attribution license which permits unrestricted use, distribution, and reproduction in any medium, provided the original work is properly cited. 